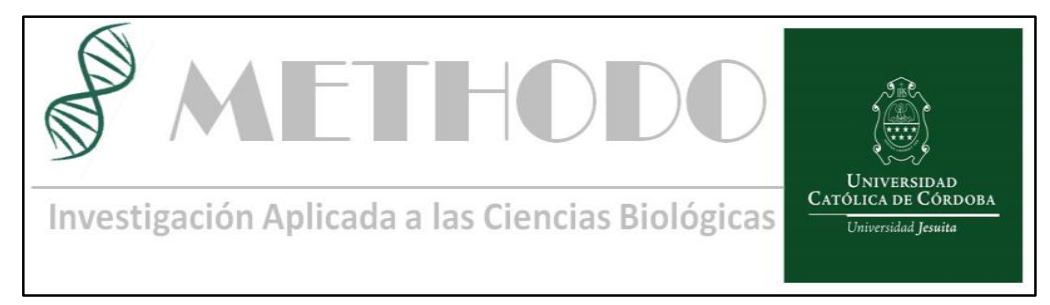

\title{
Dolor y complicaciones posquirúrgicas en histerorrafia intra vs extrabdominal en operación cesárea
}

\section{Pain and post-surgical complications of intra vs extrabdominal hysterorrhaphy in cesarean section}

\author{
Nadia Alesso ${ }^{1}$, Ariel Bertoldi ${ }^{2}$ \\ 1 Servicio de Medicina Interna Sanatorio Privado de la Cañada Córdoba Argentina. \\ 2. Servicio de Ginecología de la Clínica Universitaria Reina Fabiola. \\ Correspondencia: Nadia Alesso Servicio de Medicina Interna 1248 -X5004FHP-Córdoba, Argentina; e-mail:lulitorres_12@hotmail.com.
}

\section{Resumen}

INTRODUCCIÓN: La cesárea es la intervención quirúrgica que tiene como objetivo extraer el producto de la concepción y sus anexos ovulares a través de una laparotomía e incisión de la pared uterina. Existen varias técnicas de cesárea con diferentes resultados en dolor postoperatorio, pérdida sanguínea, duración de la intervención quirúrgica y adherencias en las siguientes cirugías.

MATERIALES Y METODOS: Estudio observacional, prospectivo y analítico. La población estuvo constituida por casos consecutivos de pacientes sometidas a cesárea, desde $1^{\circ}$ de julio del 2017 al $10^{\circ}$ de enero del 2018 en la Clínica Universitaria Reina Fabiola, las cuáles fueron valoradas de acuerdo al tipo de histerorrafia durante las primeras 24 horas del puerperio y en el puerperio tardío (10 días) en relación a las siguientes variables: dolor, rescates analgésicos, complicaciones postquirúrgicas (náuseas, vómitos, anemia) y tiempo quirúrgico.

OBJETIVOS: Comparar el dolor postquirúrgico y la necesidad de rescates analgésicos, las complicaciones postquirúrgicas y la duración de la cirugía en pacientes sometidas a operación cesárea con histerorrafia intra-abdominal versus extra-abdominal.

RESULTADOS: Este estudio incluyó un total de 200 mujeres; 100 correspondientes al grupo 1 (histerorrafia intrabdominal In situ) y 100 pertenecientes al grupo 2 (histerorrafia extrabdominal exteriorización). Las pacientes del grupo 1 presentaron menor intensidad de dolor a las 12 horas (Mediana= 5.1, $\mathrm{RIC}=3.5$ vs Mediana=6,0 $\mathrm{RIC}=3.2 ; \mathrm{p}=0,01)$, a las 24 horas $($ Mediana $=3.1, \mathrm{RIC}=4$ vs Mediana= 4.5, $\mathrm{RIC}=4 ; \mathrm{p}=0,01)$ y a los 10 días (Mediana $=1, \mathrm{RIC}=2$ vs Mediana $=2, \mathrm{RIC}=2 ; \mathrm{p}=<<0,01$ ), menor tiempo quirúrgico (Media 39,4 minutos vs media 51,1 minutos $\mathrm{p}=<<0.01$ ) y anemia $(20.99 \%$ vs 37,21\%, $\mathrm{p}=0,033)$. No hubo diferencias en relación al número de rescates analgésicos ni complicaciones postquirúrgicas tales como náuseas ni vómitos.

CONCLUSION: En este estudio, las pacientes sometidas a operación cesárea con histerorrafia intrabdominal presentaron menor dolor, menor frecuencia de anemia y tiempo quirúrgico en comparación con las pacientes en las que se realiza histerorrafia extra-abdominal. No se encontraron diferencias, sin embargo, en el número de rescates analgésicos y en la frecuencia de complicaciones post-operatorias tales como náuseas ni vómitos.

Palabras claves: operación cesárea, tipo de histerorrafia, dolor postquirúrgico, complicaciones postoperatorias.

Revista Methodo: Investigación Aplicada a las Ciencias Biológicas. Facultad de Medicina. Universidad Católica de Córdoba. Jacinto Ríos 571 Bo Gral. Paz. X5004FXS. Córdoba. Argentina. Tel.: (54) 3514517299 / Correo: methodo@ucc.edu.ar / Web: methodo.ucc.edu.ar| ARTICULO ORIGINAL Methodo 2019;4 (1):06-11 


\section{Abstract}

INTRODUCTION: Cesarean section is the surgical intervention that aims to extract the product of conception and its ovular attachments through a laparotomy and incision of the uterine wall. There are several cesarean techniques with different results in postoperative pain, blood loss, duration of surgery and adhesions in the following surgeries.

MATERIALS AND METHODS: Observational, prospective and analytical study. The population consisted of consecutive cases of patients undergoing caesarean section, from July 1 st, 2017 to January 10th, 2018 at the Clinica Universitaria Reina Fabiola, which were assessed according to the type of hysterorraphy during the first 24 hours of the puerperium and in the late puerperium (10 days) regarding to the following variables: pain, analgesic rescues, postsurgical complications (nausea, vomiting, anemia) and surgical time. OBJETIVE: To compare postoperative pain and the need for analgesic rescues, postsurgical complications and the duration of surgery in patients undergoing cesarean section surgery with intra-abdominal versus extra-abdominal histerorrhaphy.

RESULTS: This study included a total of 200 women; 100 corresponding to group 1 (intra-abdominal histerorrhaphy In situ) and 100 belonging to group 2 (extra-abdominal histerorrhaphy exteriorización). Patients in group 1 had a lower intensity of pain at 12 hours (Median $=5.1, \mathrm{ICR}=3.5$ vs Median $=6.0 \mathrm{ICR}$ $=3.2, \mathrm{p}=0.01$ ), at 24 hours (Median $=3.1, \mathrm{ICR}=4 \mathrm{vs}$ Median $=4.5, \mathrm{RIC}=4, \mathrm{p}=0.01$ ) and at 10 days (Median $=1$, RIC $=2$ vs Median $=2$, RIC $=2, p=<<0.01$ ), shorter surgical time ( Mean 39.4 minutes vs mean 51.17 minutes $\mathrm{p}=<<0.01)$ and anemia $(20.99 \%$ vs $37.21 \%, \mathrm{p}=0.033)$. There were no differences in relation to the number of analgesic rescues or postoperative complications such as nausea or vomiting. CONCLUSION: In this study, patients undergoing cesarean section with intra-abdominal hysterorrhaphy presented less pain, less frequency of anemia and surgical time compared with patients in whom extraabdominal hysterorrhaphy was performed. No differences were found, however, in the number of analgesic rescues and in the frequency of postoperative complications such as nausea or vomiting.

Keywords: cesarean section, type of hysterorrhaphy, postoperative pain, post-operative complications.

\section{Introducción}

La cesárea es la intervención quirúrgica que tiene como objetivo extraer el producto de la concepción y sus anexos ovulares a través de una laparotomía e incisión de la pared uterina ${ }^{1}$.

Más allá de los avances que se han hecho en el conocimiento de la fisiopatología del dolor y sus consecuencias, muchas encuestas señalan que el dolor postoperatorio es un flagelo prevalente en los pacientes quirúrgicos y las cesáreas no escapan a dicha realidad ${ }^{2}$. Las causas son múltiples y variadas: falta de conocimiento por parte del personal médico, factores culturales, escasez de recursos, entre otros ${ }^{3-4}$. Uno de los factores relacionados al dolor postoperatorio de la cesárea es la técnica quirúrgica utilizada.

Las técnicas quirúrgicas varían considerablemente, dependiendo de la situación clínica y las preferencias del cirujano. Existen varias técnicas de cesárea: Cesárea Pfannenstiel, Cesárea tipo Pelosi, Técnica de Joel Cohen, Técnica de Misgav-Ladach, Misgav-Ladach modificada y la cesárea tradicional (incisión mediana baja) ${ }^{5-10}$.

Los estudios disponibles sugieren que las técnicas de Joel-Cohen y Misgav-Ladach modificadas (histerorrafia intrabdominal) tienen ventajas por encima de las técnicas Pfannenstiel y tradicional (histerorrafia extrabdominal): menos dolor postoperatorio, menor necesidad de analgesia, reducción de la pérdida sanguínea, menor duración de la intervención quirúrgica, menos adherencias en las siguientes cirugías ${ }^{5-10}$. El control del dolor de una paciente cursando el postoperatorio inmediato de una cesárea supone desafíos y beneficios únicos e importantes ${ }^{11}$. Dichos pacientes tienen riesgo incrementado de fenómenos tromboembólicos, los cuales pueden estar favorecidos por falta de movilización activa; deben, además, alimentar al recién nacido, quien indirectamente puede verse expuesto a los fármacos que le sean administrados a la madre. Por otro lado, estas mujeres deben estar alertas y en condiciones de amamantar a sus recién nacidos ${ }^{12}$. De esta forma el analgésico elegido debe de tener baja transferencia a la leche materna, efecto mínimo o nulo en el neonato y en la madre, asegurando analgesia y un alta temprana.

Es indiscutible que las técnicas que se asocian a menor dolor y pérdida sanguíneas, se relacionan con mejor confort postquirúrgico, lo cual genera beneficios para el alta hospitalaria temprana y el reinicio de las actividades. 


\section{Objetivos}

Principal: Comparar el dolor postquirúrgico y la necesidad de rescates analgésicos en pacientes sometidas a operación cesárea con histerorrafia intra-abdominal versus extra-abdominal.

Secundario: Comparar las complicaciones postquirúrgicas (náuseas, vómitos, anemia) y la duración del tiempo quirúrgico en pacientes sometidas a operación cesárea con reparación uterina intra-abdominal versus extra-abdominal.

\section{Pacientes y métodos}

Diseño: Estudio observacional, prospectivo, analítico.

Población: La población estuvo constituida por casos consecutivos de pacientes sometidas a cesárea, desde $1^{\circ}$ de julio del 2017 al $10^{\circ}$ de enero del 2018 en la Clínica Universitaria Reina Fabiola (CURF), las cuáles fueron valoradas de acuerdo al tipo de histerorrafia durante las primeras 24 horas del puerperio (puerperio inmediato) y en el puerperio tardío (10 días) en relación a las siguiente variables: dolor, rescates analgésicos, complicaciones postquirúrgicas (náuseas, vómitos, anemia) y tiempo quirúrgico. Muestra: Técnica de muestreo:

Grupo 1 (histerorrafia intra-abdominal): Todas las cesáreas con histerorrafia intrabdominal.

Grupo 2 (histerorrafia extra-abdominal): Primer cesárea con histerorrafia extrabdominal a continuación de una paciente sometida a cesárea del grupo 1.

Criterios de inclusión:

- Embarazadas sometidas a cesárea, que ingresaron a la Clínica Universitaria Reina Fabiola durante el período comprendido entre el 1 de julio del 2017 hasta el 10 de enero de 2018.

Criterios de exclusión:

Incisiones verticales uterinas

Historia de abuso de opiáceos

Anestesia general

Alergia a los AINES

Cesáreas más complicaciones intraquirurgicas (histerectomías, cistorrafias, etc.)

- Cesáreas más cirugías agregadas (ligadura tubaria bilateral, quistectomía, hernioplastia, etc.)

Variables de estudio:

Cuantitativas:

$\begin{array}{ll}\text { - } & \text { Edad de la paciente } \\ \text { - } & \text { Peso } \\ \text { - } & \text { Talla } \\ \text { - } & \text { IMC }\end{array}$

\section{Edad gestacional}

- Número de gestas

- Duración de la cirugía (en minutos): se realizó una división categórica, dividiéndolo en cuatro grupos: menos de 30 minutos, entre 30 y menos de 45 minutos, entre 45 y 60 minutos y aquellas cirugías que duraron más de 60 minutos, desde el inicio de la incisión en piel

- Escala del dolor: Escala visual análoga (EVA): la intensidad del dolor se representó en una línea de $10 \mathrm{~cm}$. En uno de los extremos constaba la frase de "no dolor" y en el extremo opuesto "el peor dolor imaginable". La distancia en centímetros desde el punto de «no dolor» a la marcada por el paciente representó la intensidad del dolor. La paciente marcó con una línea la intensidad del dolor a las 6,12 y 24 horas postcesárea (puerperio inmediato). La valoración del dolor a los 10 días postquirúrgicos, se realizó a través de una llamada telefónica.

- Rescate analgésico (cantidad de rescates) a las 6, 12, 24 hs postquirúrgicas.

Cualitativas:

- Náuseas

- Vómitos

- Anemia: definida por un valor de hemoglobina $<12 \mathrm{~g} / \mathrm{dl}$ y consignada al ingreso de la institución, la cual es solicitada en el laboratorio pre-quirúrgico y a los 10 días del postquirúrgico.

- Otras incisiones quirúrgicas

- Tipo de gestas previas: Partos-CesáreasAbortos- Embarazo ectópico.

El rescate analgésico que se realiza en el Servicio de Tocoginecología de la Institución se encuentra protocolizado.

Todas estas variables fueron recabadas en hojas de captura de datos diseñada para este fin.

Consideraciones éticas:

El protocolo de estudio fue evaluado y aprobado por el Comité de Ética e Investigación de la Clínica Universitaria Reina Fabiola. El mismo se rigió bajo las normas de la Declaración de Helsinki (2008), la ley de confidencialidad de datos personales (25.326), el sistema de evaluación, registro y fiscalización de las investigaciones en salud (ley 9694) de la Pcia. De Córdoba y la Guía de Buenas Prácticas Clínicas en Investigación en Salud de la ANMAT.

Previo a la obtención de cualquier dato relacionado con el estudio, las pacientes fueron informadas oralmente y por escrito sobre todos los aspectos del estudio de investigación a través de una hoja de consentimiento informado, el cual fue firmado por las pacientes. 
Análisis estadístico: Para comparar la severidad de dolor se utilizó el test de Wilcoxon. Para comparar la necesidad de rescates analgésicos, así como la frecuencia de complicaciones postquirúrgicas y el tiempo quirúrgico se utilizó el test de Chi cuadrado.

\section{Resultados}

El análisis de este estudio incluyó un total de 200 mujeres; 100 correspondientes al grupo 1 (histerorrafia intrabdominal) y 100 pertenecientes al grupo 2 (histerorrafia extrabdominal). En la Tabla 1, se muestran las características demográficas de las pacientes. Los dos grupos fueron homogéneos con respecto a dichas variables. La anestesia utilizada en todos los casos fue la raquídea. La analgesia posoperatoria fue de rutina en todas las pacientes (Apéndice A). Las indicaciones de la operación cesárea se señalan en la Tabla 2.

Tabla 1: Características de las pacientes estudiadas de acuerdo a la histerorrafia realizada.

\begin{tabular}{|c|c|c|}
\hline & $\begin{array}{l}\text { In situ } \\
(n=100)\end{array}$ & $\begin{array}{l}\text { Exteriorización } \\
(n=100)\end{array}$ \\
\hline Edad (años) ${ }^{*}$ & $32.7+/-5$ & $32.27+1-5$ \\
\hline Peso $(\mathrm{K})^{*}$ & $75.43+/-14$ & $75.52+/-14$ \\
\hline Talla $(m)^{\star}$ & $1.62+1-0.1$ & $1.63+/-0.1$ \\
\hline Edad gestacional (semanas)* & $38.64+1-2$ & $39.01+/-1$ \\
\hline Hemoglobina pre quirúrgica $(\mathrm{g} / \mathrm{d} \mathrm{d})^{*}$ & $12.02+/-1$ & $11.76+/-1$ \\
\hline Nuligestas & 42 & 48 \\
\hline Gestas previas & 58 & 52 \\
\hline Cesáreas previas & 47 & 50 \\
\hline Una cesárea previa & 42 & 39 \\
\hline Dos cesáreas previas & 5 & 11 \\
\hline Abortos & 7 & 2 \\
\hline Partos previos & 5 & \\
\hline
\end{tabular}

Tabla 2: Indicación de operación cesárea (\%) en la muestra de pacientes estudiadas.

\begin{tabular}{lr}
\hline Cesárea previa & 39 \\
Falta de progreso & 17,5 \\
Podálico & 8,5 \\
Dos cesáreas previas & 8,5 \\
Desproporción & 6,5 \\
Sufrimiento fetal agudo & 6,5 \\
Falta de descenso & 4 \\
Electiva & 4 \\
Gemelar & 2 \\
Placenta previa & 2 \\
Otras & 3,5 \\
\hline
\end{tabular}
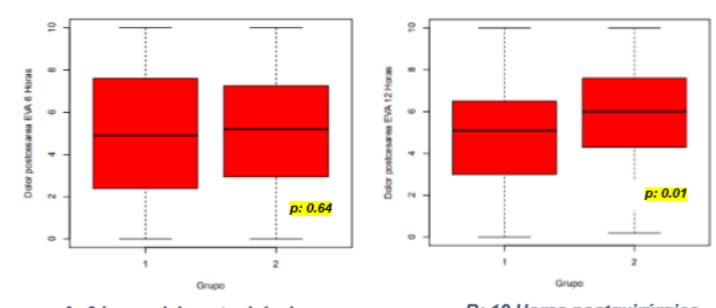

A: 6 horas del postquirúrgico

B: 12 Horas postquirúrgico
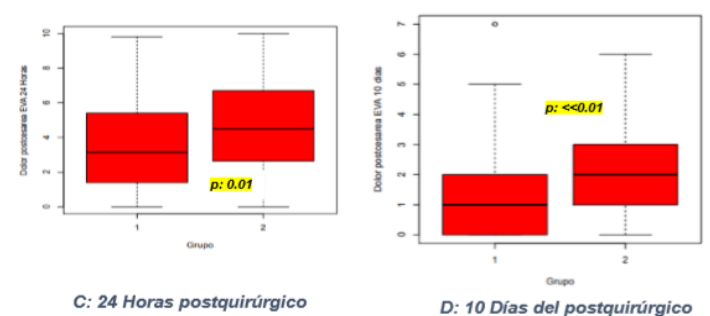

Figura 1: Comparación entre ambos grupos de la escala visual análoga EVA en el postquirúrgico. Ilustra la intensidad del dolor en ambos grupos de pacientes a las $6,12,24$ horas del postquirúrgico y a los 10 días. Como se observa, no se objetivaron diferencias significativas entre ambos grupos de pacientes en el postoperatorio temprano (6 horas); sin embargo, las pacientes con histerorrafia intra-abdominal presentaron menor intensidad de dolor a las 12 horas (Mediana=5.1, $\mathrm{RIC}=: 3.5$ vs Mediana $=6, \mathrm{RIC}=3.2$ ), a las 24 horas (Mediana $=3.1, \mathrm{RIC}=4$ vs Mediana $=4.5$, $\mathrm{RIC}=4)$ y a los 10 días (Mediana $=1, \mathrm{RQ}=2$ vs Mediana $=2$, $\mathrm{RIC}=2$ ).

En relación al tiempo quirúrgico, como se observa en la Tabla 3, la mayoría de las cirugías en el grupo 1 histerorrafia intra-abdominal-In situ fueron finalizadas dentro de los 45 minutos, a diferencia del grupo 2 histerorrafia extraabdominal -exteriorización, que el $71 \%$ fueron finalizadas entre los 45 y 60 minutos ( $p=$ $<<0.01)$. Con respecto a la anemia, solo se realizaron el control de hemoglobina a los 10 días del postoperatorio en 81 pacientes del grupo $1 \mathrm{y}$ 86 pacientes del grupo 2; observándose una menor frecuencia de anemia en el grupo $1(20,99$ $\%$ del grupo 1 vs $37,21 \%$ del grupo $2 ; \mathrm{p}=0.033$ ) (Tabla 3). No se encontraron diferencias entre ambos grupos en los siguientes resultados: número de rescates analgésicos, frecuencia de náuseas $(\mathrm{p}=0,501)$ y vómitos. Tabla 3 y 4

Tabla 3: Tiempo quirúrgico, Anemia y Nauseas

\begin{tabular}{|c|c|c|c|c|c|c|}
\hline \multirow[t]{2}{*}{$\%$} & \multicolumn{4}{|c|}{ Tiempo quirúrgico } & \multirow[t]{2}{*}{ Anemia } & \multirow[t]{2}{*}{ Nauseas } \\
\hline & $<30$ & $30-<45$ & $45-60$ & $>60$ & & \\
\hline In situ & 11 & 59 & 26 & 4 & 20,99 & 2 \\
\hline Exteriorización & 1 & 28 & 62 & 9 & 37,21 & 0 \\
\hline valor $p$ & \multicolumn{4}{|c|}{$<<0,01$} & 0.033 & 0.501 \\
\hline
\end{tabular}

Revista Methodo: Investigación Aplicada a las Ciencias Biológicas. Facultad de Medicina. Universidad Católica de Córdoba. Jacinto Ríos 571 Bo Gral. Paz. X5004FXS. Córdoba. Argentina. Tel.: (54) 351 4517299 / Correo: methodo@ucc.edu.ar / Web: methodo.ucc.edu.ar| ARTICULO ORIGINAL Methodo 2019;4 (1):06-11 
Tabla 4: Numero de recates analgésicos en el postquirúrgico.

\begin{tabular}{c|rrr|rrr|rrr}
$\%$ & \multicolumn{3}{|c|}{6 horas } & \multicolumn{3}{|c|}{12 horas } & \multicolumn{3}{|c}{24 horas } \\
\hline $\begin{array}{c}\text { Numero de } \\
\text { rescates }\end{array}$ & 0 & 1 & 2 & 0 & 1 & 2 & 0 & 1 & 2 \\
In situ & 64 & 35 & 1 & 71 & 28 & 1 & 89 & 9 & 2 \\
Exteriorizacion & 63 & 37 & 0 & 70 & 26 & 4 & 87 & 13 & 0 \\
valor $p$ & & 0.886 & & & 0.452 & & \multicolumn{3}{|c}{0.296} \\
\hline
\end{tabular}

\section{Discusión}

La técnica quirúrgica ideal para realizar la operación cesárea continúa generando debate entre la comunidad de obstetras, particularmente debido a un incremento del procedimiento a nivel global.

Numerosos aspectos técnicos se han discutidos incluyendo la incisión, el método de alumbramiento, el cierre o no del peritoneo y el material de sutura, entre otros. Sin embargo, lo que genera un conflicto por sus diversas opiniones es el cierre del útero intrabdominal versus extrabdominal ${ }^{1-14}$. En nuestro estudio, se evaluaron solamente los resultados con reparación intrabdominal y extrabdominal, haciendo hincapié en el dolor postquirúrgico, tiempo quirúrgico, numero de rescates analgésicos, anemia y nauseas. Algunos estudios previos no demuestran diferencias significativas a nivel global entre estas dos opciones, excepto en la duración de la cirugía y el número de suturas utilizadas, éste parámetro no fue evaluado en nuestro trabajo ${ }^{14}$ Nuestros hallazgos revelan diferencias estadísticamente significativas con respecto al dolor postquirúrgico a partir de las 6 horas del postoperatorio, resultados que se repiten para las 12 y 24 horas posteriores a la operación y a los 10 días de la cirugía, entre ambos grupos, a favor de la reparación uterina intrabdominal. Estos resultados coinciden con publicaciones, que describen las ventajas que existen entre reparar el útero de forma in situ versus la exteriorización del mismo, como menor dolor, menor necesidad de analgésicos, menor pérdida de sangre, duración quirúrgica, a favor de la reparación intrabdominal. .-10 $^{5}$

En nuestro estudio podemos observar que más del $70 \%$ de las pacientes con reparación intrabdominal tuvieron un tiempo quirúrgico menor de los 45 minutos, mientras que solo el $29 \%$ de las pacientes con reparación extrabdominal obtuvieron dicho tiempo, cabe destacar que todas las operaciones cesáreas, empleando ambas técnicas quirúrgicas, fueron realizadas por diferentes cirujanos, y que en el $100 \%$ de los casos participaron médicos en periodo de formación, anulando así el factor humano con respecto al tiempo quirúrgico. Es conocido en diferentes estudios publicados, el manejo de la seguridad del paciente quirúrgico, dentro de estos parámetros el beneficio del menor tiempo quirúrgico en las cirugías a cielo abierto, como aquel relacionado a los procesos infecciosos. ${ }^{15}$

Con respecto a la anemia, también se observó con menor frecuencia en el grupo de reparación intrabdominal, pero al igual que en otras publicaciones no fue necesario el tratamiento transfusional en ningún paciente, independientemente del tipo de histerorrafia practicada.

En relación a la necesidad de rescates analgésicos y en disidencia con otros estudios, no hubo diferencias significativas entre ambos grupos; suponemos que existe un factor de subjetividad entre la valoración del dolor y los rescates analgésicos que fueron necesarios, lo que explicaría la diferencia significativa que, si se presentó en la escala del dolor postquirúrgico, pero no así en el número de rescates analgésicos. En muchos estudios, se relaciona la reparación uterina exteriorizada con mayor incidencia de náuseas, vómitos; no fue el caso de nuestro estudio, donde sí se observó un $2 \%$ de nauseas en el grupo de reparación intrabdominal, aunque esta diferencia no fue significativa.

\section{Conclusión}

Este estudio demostró que las pacientes sometidas a operación cesárea con histerorrafia intrabdominal presentaron menor dolor, menor frecuencia de anemia y tiempo quirúrgico. Sin embargo, ambas técnicas de histerorrafia impresionan ser equivalentes en términos de necesidad de analgesia, náuseas y vómitos.

\section{Bibliografía}

1. Cunningham, G.F., Mac Donald, C.P., Gant, F.N., Leveno, J.K., Gilstrap, C.L., Hankins, D.V. and Clark, C.S. Sección IV: Capitulo 25: Cesárea. Williams Obstetricia. 24 ${ }^{\mathrm{a}}$. Editorial MCGRAWHILL. 2015. 534. 
2. Villar J, Valladares E, Wojdyla D, Zavaleta N, Carroli G, Velazco A, et al. Caesarean delivery rates and pregnancy outcomes: The 2005 WHO global survey on maternal and perinatal health in Latin America. Lancet. 2006; 367:1819-29.

3. Apfelbaum JL, Chen C, Mehta SS, Gan TJ. Postoperative pain experience: Results from a national survey suggest postoperative pain continues to be undermanaged. Anesth Analg. 2003; 97:534-40.

4. Rawal N. 10 years of acute pain services: Achievements and challenges. Reg Anesth Pain Med. 1999; 24:68-73.

5. Hofmeyr GJ, Mathai M, Shah AN, et al. Techniques for caesarean section. Cochrane database of systematic reviews 2008, Issue 1. Art. No: CD004662.

6. Xavier P, Ayres-De-Campos D, Reynolds A, et al. The modified Misgav-Ladach versus the Pfannenstiel-Kerr technique for cesarean section: A randomized trial. Acta Obstet Gyn Scand 2005; 84:878-882.

7. Abalos E. Técnicas quirúrgicas para la cesárea: Comentario de la BSR (última revisión: 1 de mayo de 2009). La Biblioteca de Salud Reproductiva de la OMS; Ginebra: Organización Mundial de la Salud.

8. Orji EO, Olaleye AO, Loto OM, et al. A randomized controlled trial of uterine exteriorization and non-exteriorization at cesarean section. Aust N Zel Obstet Gynaecol 2008; 48:570-574.

9. David Alejandro Martínez Ceccopieri, Ernesto Barrios Prieto, David Martínez Ríos. Cesarea Misgav-Ladach modificada en un Hospital de tercer nivel. Ginecol Obstet Mex 2012; 80:501-508

10. S. Nafisi. Influence of uterine exteriorization versus in situ repair on post-cesarean maternal pain: a randomized trial. 2007; 16:135-8.

11. Samina Ismail, Khurram Shahzad, and Faraz Shafiq Observational study to assess the effectiveness of postoperative pain management of patients undergoing elective cesarean section. J Anaesthesiol Clin Pharmacol. 2012; 28: 36-40.

12. A. Faboya, D. Uncles. Post cesarean delivery pain management: Multimodal approach. Int J Obstet Anesth. 2006; 15:185-8.

13. Asokumar Buvanendran, MD. Multimodal Analgesia for Perioperative Pain Management. American Society of Anesthesia consult material. Acceso en http: // www.asa.com. Último acceso (julio/2016)

14. I. Cristina, MD, M. Ramos de Amorim, MD. Uterine exteriorization Compared with in situ repair at cesarean delivery. 2008.

15. J. Carlos Meneu Díaz. Predictores de complicaciones en cirugía. Seguridad del paciente quirúrgico. OMS 2017.

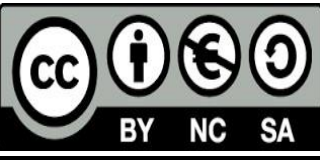

Revista Methodo: Investigación Aplicada a las Ciencias Biológicas. Facultad de Medicina. Universidad Católica de Córdoba. Jacinto Ríos 571 Bo Gral. Paz. X5004FXS. Córdoba. Argentina. Tel.: (54) 351 4517299 / Correo: methodo@ucc.edu.ar / Web: methodo.ucc.edu.ar| ARTICULO ORIGINAL Methodo 2019;4 (1):06-11 